\section{The ecological solution}

\author{
Alex Kacelnik
}

Dynamic Modeling in Behavioral Ecology. By Marc Mangel and Colin W. Clark. Princeton University Press: 1989. Pp. 308. Hbk \$45, £28; pbk \$15.95, £10.

Stochastic dynamic programming (SDP) is a technique to find optimal solutions when the payoff of alternative actions is probabilistic, conditional on the state of the optimizing agent and dependent on the time of the action. It has been used in behavioural ecology for more than a decade, and in other fields for much longer. So can there be much excitement about a new book on the subject? In this case, the answer is 'yes'. Mangel and Clark's volume combines the clarity of an extremely pedagogical - indeed at times even patronizing - textbook with insightful accounts of the application of SDP to a variety of biological problems.

The section on fundamentals, in spite of requiring little previous mathematical knowledge, succeeds well in describing the probability concepts needed to tackle a broad range of stochastic problems. The authors even explain how to write a computer program. This section will show those biologists who claim that they cannot get involved in formal modelling, because of a lack of expertise in mathematics, how wrong they are. It also includes a discussion of the classical optimal-foraging problem of patch selection, treated in the framework of SDP. Without explaining the principles, the authors lead the reader gently through implementation of a full SDP model. People familiar with state-independent, rate-maximizing solutions will be impressed by the understanding that this simple model provides.

The core of the book describes applications of SDP to various biological problems, including hunting in lions, reproductive strategies in insects, vertical migrations of plankton and planktivorous animals, parental care in birds, and dispersal in spiders and raptors. Each example is clearly presented and carefully chosen to introduce a new awareness of the resources available to the modeller. Although the intention is to provide an introduction to the models, not to justify each one in detail, and although much of the material has been presented elsewhere, this section includes many novel additions and in itself contributes substantially to the various areas of research involved.

Treatment of the principles and extensions of SDP follows the applications in the core of the book. This may seem an unusual sequence, but it works well. Instead of being over-loaded with information before its possible uses are made clear, the reader is enabled to appreciate the potential applications of each method by generalization from particular cases.

As the authors explicitly point out, SDP is not the only way of tackling problems of optimality in behavioural ecology. In an effort to be fair, they include a brief discussion of other methods, but their sympathies are obvious and one by one other techniques are shown to be less promising than SDP. Newcomers to modelling, however, should be made aware that other approaches can, in some circumstances, be preferable. For example, solutions obtained by SDP usually (but not only) come as tables showing what the agent must do if a

\section{A light touch}

\section{S. D. Smith}

Optical Computing: A Survey for Computer Scientists. By Dror G. Feitelson. MIT Press: 1988. Pp.393. \$37.50, £29.95.

ThIs is a remarkable book. Written by a computer scientist, it deals with a subject that is currently only a research topic and that spans several disciplines in optical physics and information technology. Essentially it is a survey of the field written by an outsider for outsiders; but such is its quality that in my opinion it should be standard reading for researchers themselves.

Feitelson begins with a short introduction, making a few general points about why optics might be interesting in the field of computing, and giving some guidance to relevant journals. He then continues with an unexceptional but well-written 50-page background account of optics.

A comprehensive chapter on optical image and signal processing follows which is well placed in the context of the subject. The author clearly realizes the importance of harnessing the two-dimensional capability of optics. He provides excellent descriptions of optical Fourier transform procedures and pattern recognition, including cross correlation, optical frequency plane correlators and matched filtering techniques. This topic is thoroughly treated, and the chapter extends to synthetic aperture radar imaging and radio signal analysis. Optical processing technology has, of course, had some success in practical systems for these applications, but we still await more powerful real-time technology.

The fourth chapter deals with numerical processing by optical methods, and is a useful listing. Feitelson briefly considers the combination of optical and electronic number of conditions are met. These tables tend to be rather unwieldy when the main interest is the link between optimal behaviour and psychological or physiological mechanisms; although alternative approaches such as control systems theory are not as user-friendly when one is calculating optimal solutions, they do make it easier to cross levels of integration.

This is not a criticism of Mangel and Clark, who are mostly involved with optimality problems; rather it is a note of caution which I voice because of the very success that I expect and wish for this excellent book. It should have a considerable influence on the spread of theoretical modelling in behavioural ecology.

Alex Kacelnik is in the Biological Information Processing Group, King's College, Cambridge, CB2 1ST, UK. systems by describing not only what optics can do for computing but also what electronics can do for optical processing. His remarks about the value of electronic control of optical logic planes are to the point, but he seems to have missed the concept of a 'lock and clock' architecture, which is necessary to protect information transmitted at the speed of light from being corrupted. The review of the interaction of nonlinear optics on possible future digital optical technology specifically excludes any discussion of the physical mechanisms of such nonlinearities. Because it is these mechanisms that control switching energies and operating powers, the author restricts himself to reporting the literature as read.

The state of research is faithfully represented, however, although I would like to have seen some question marks raised over the small amount of existing experimental work on digital optical circuits that which has been done has shown that indefinitely extensible restoring optical logic is practicable. The general importance of the spatial light modulator and its digital equivalent, the optical logic plane, comes through well.

The book ends with a sensible discussion of the place and role of optical computing, some useful appendices and a large and up-to-date reference list. Altogether, Feitelson has written a most useful volume which deserves wide circulation.

S.D. Smith is a Professor in the Department of Physics, Heriot-Watt University, Riccarton, Edinburgh EH14 4AS, UK, and co-founder of Edinburgh Instruments.

\section{Second editions}

- Earth and Life Through Time by Steven M. Stanley. Publisher is W. H. Freeman, price is hbk $\$ 39.95, £ 31.95$, pbk $\$ 26.95, £ 18.95$. For review see Nature 319, 811 (1986)

- An Introduction to Chaotic Dynamical Systems by Robert L. Devaney. Publisher is Addison-Wesley, price is $£ 34.95$. 